\title{
Determining pure discrete spectrum for some self-affine tilings
}

\author{
Shigeki Akiyama1" \\ Franz Gähler" \\ Jeong-Yup Lee 3 \\ ${ }^{1}$ Institute of Mathematics, University of Tsukuba, Japan \\ ${ }^{2}$ Faculty of Mathematics, Bielefeld University, Germany \\ ${ }^{3}$ Dept. of Math. Edu., Catholic Kwandong University, Republic of Korea \\ received $4^{\text {th }}$ Apr. 2014, revised $2^{\text {nd }}$ Oct. 2014, accepted $13^{\text {th }}$ Oct. 2014.
}

By the algorithm implemented in the paper by Akiyama-Lee [Adv. Math. 226(4):2855-2883, 2011] and some of its predecessors, we have examined the pure discreteness of the spectrum for all irreducible Pisot substitutions of trace less than or equal to 2, and some cases of planar tilings generated by boundary substitutions due to Kenyon [Geom. Func. Anal. 6:471-488, 1996].

Keywords: Pisot substitution, pure discrete spectrum, self-affine tiling

\section{Introduction}

Self-affine tilings are often studied as examples of tiling dynamics. Many equivalent conditions are known for the spectrum of the tiling dynamics to be pure discrete [4], but none of them is known to hold in general. For particular instances of tilings, there are algorithms by which one can check whether the spectrum is pure discrete. The overlap algorithm [20] and the balanced pair algorithm [20] are practically usable mostly in one dimension, but the potential overlap algorithm of Akiyama and Lee [3] is of practical use in all dimensions, even if the tiles have complicated geometries. Here we use these algorithms to check the pure discreteness of the spectrum of the tiling dynamics for special cases of self-affine tilings. One of these cases is the 1-dimensional irreducible Pisot substitution tilings. There is a long-standing conjecture [4] that these tilings have pure discrete spectrum. For the cases with two tiles, it is known that the conjecture is true [12], but not much is known for more than two tiles. Already the case with three tiles is computationally involved. We concentrate here on the cases with substitution matrices of small trace. More precisely, all inequivalent substitution matrices $M$ with $\operatorname{Tr}(M) \leq 2$ have been generated, and for all substitutions with these matrices (446683 substitutions in total), we have checked that the spectrum is pure discrete.

\footnotetext{
*akiyama@math.tsukuba.ac.jp

†gaehler@math.uni-bielefeld.de

$\ddagger$ jylee@cku.ac.kr. Corresponding author
} 
The other type of tilings we consider are the self-affine tilings constructed from the endomorphisms of free groups by Kenyon [14]. We have looked at the cubic polynomials whose coefficients are all less than or equal to 3, except for a single case, whose computation is beyond our computer capability. All other examples turn out to be pure discrete.

We also give some non pure discrete examples of self-affine tilings in 4 . The construction of the first one is due to Bandt [6]. The second arises from 4 interval exchanges studied by Arnoux-Ito-Furukado. Both satisfy the Pisot family condition, so that their translation actions are not weakly mixing. We provide the programs in [2, 10].

\section{Pisot substitutions with small trace}

In this section, we wish to computationally confirm the Pisot substitution conjecture to be true for a class of simple, irreducible Pisot substitutions with three tiles. Consider a monoid $\mathcal{A}^{*}$ over finite alphabets $\mathcal{A}$ equipped with concatenation and write the identity as $\epsilon$, the empty word. A symbolic substitution $\sigma$ is a non-erasing homomorphism of $\mathcal{A}^{*}$, defined by $\sigma(a) \in \mathcal{A}^{+}=\mathcal{A}^{*} \backslash\{\epsilon\}$ for $a \in \mathcal{A}$. The set $\mathcal{A}^{\mathbb{Z}}$ of two sided sequences is compact by the product topology of the discrete topology on $\mathcal{A}$. The substitution $\sigma$ acts naturally on $\mathcal{A}^{\mathbb{Z}}$ by $\sigma\left(\ldots a_{-1} a_{0} a_{1} a_{2} \ldots\right)=\ldots \sigma\left(a_{-1}\right) \sigma\left(a_{0}\right) \sigma\left(a_{1}\right) \sigma\left(a_{2}\right) \ldots$ Let $M_{\sigma}$ be the incidence matrix $\left(|\sigma(j)|_{i}\right)_{i j}$ where $i, j \in \mathcal{A}$. Here $|w|_{j}$ is the cardinality of $j$ appearing in a word $w \in \mathcal{A}^{*}$. Denote by $\chi_{\sigma}$ the characteristic polynomial of $M_{\sigma}$. The substitution $\sigma$ is primitive if $M_{\sigma}$ is primitive and it is irreducible if $\chi_{\sigma}$ is irreducible ${ }^{(i)}$ A Pisot number is an algebraic integer $\lambda>1$ whose all the other algebraic conjugates of $\lambda$ lie strictly inside the unit circle. If the Perron-Frobenius root of $M_{\sigma}$ is a Pisot number then we say that $\sigma$ is a Pisot substitution. A word $w \in \mathcal{A}^{*}$ is admissible if there exist $k \in \mathbb{N}$ and $a \in \mathcal{A}$ such that $w$ is a subword of $\sigma^{k}(a)$. Let

$$
X_{\sigma}=\left\{\left(a_{n}\right)_{n \in \mathbb{Z}} \in \mathcal{A}^{\mathbb{Z}} \mid a_{k} a_{k+1} \ldots a_{\ell} \text { is admissible for all } k, \ell \text { with } k<\ell\right\} .
$$

Then $\left(X_{\sigma}, s\right)$ forms a topological dynamical system where $s$ is the shift map defined by $s\left(\left(a_{n}\right)_{n \in \mathbb{Z}}\right)=$ $\left(a_{n+1}\right)_{n \in \mathbb{Z}}$. By the primitivity, the system is minimal and uniquely ergodic with the unique invariant measure $\mu$. Therefore we can discuss the spectrum of the unitary operator $U_{\sigma}$ acting on $L^{2}\left(X_{\sigma}, \mu\right)$ for which $\left(U_{\sigma}(f)\right)(x)=f(s(x))$. The substitution $\sigma$ has pure discrete dynamical spectrum if the spectral measure associated to $U_{\sigma}$ consists only of point spectra, or equivalently, the linear span of eigenfunctions is dense in $L^{2}\left(X_{\sigma}, \mu\right)$. It is conjectured [4] that this $\mathbb{Z}$-action by $U_{\sigma}$ is pure discrete if $\sigma$ is an irreducible Pisot substitution - so called Pisot substitution conjecture. For the primitive substitution $\sigma$, we can also discuss a natural suspension of $\left(X_{\sigma}, s\right)$ by associating to each letter the length determined by the associated entries of the left eigenvector of $M_{\sigma}$. The sequence then defines a tiling of $\mathbb{R}$ with an inflation matrix $Q=(\beta)$, where $\beta$ is the Perron-Frobenius root of $M_{\sigma}$. This gives a tiling dynamical system $\left(X_{\mathcal{T}}, \mathbb{R}\right)$ which is also minimal and uniquely ergodic. It is known [7] that if $\sigma$ is an irreducible Pisot substitution, this $\mathbb{R}$-action on $\left(X_{\mathcal{T}}, \mathbb{R}\right)$ is pure discrete if and only if the $\mathbb{Z}$-action on $\left(X_{\sigma}, s\right)$ is pure discrete.

The following assertion may be known but we did not find it in the literature. It gives a bound for the number of irreducible primitive substitutions over $m$ letters.

Lemma 2.1 Let $B>0$. The cardinality of the set of primitive substitutions over $m$ letters, whose Perron Frobenius root is less than or equal to $B$, is less than $m^{m^{4} B^{2(m-1)^{2}+2}}$.

(i) We always assume the irreducibility of $M_{\sigma}$ in the sense of Perron-Frobenius theory. So the irreducibility in this article is for $\chi_{\sigma}$. 
Proof: Let $\sigma$ be a substitution over $m$ letters whose incidence matrix is $M_{\sigma}$. Then there is a positive integer $k$ that $M_{\sigma}^{k}=M_{\sigma^{k}}$ is a positive matrix. In fact, one can take $k \leq(m-1)^{2}+1$ for all primitive matrix $M_{\sigma}$ (see [11], [18]). Denote the characteristic polynomial by $\Phi_{\sigma^{k}}(x)=x^{m}-c_{m-1} x^{m-1}-$ $c_{m-2} x^{m-2}-\cdots-c_{0}$. Then we have a bound $\left|c_{m-i}\right|<\left(\begin{array}{c}m \\ i\end{array}\right) \beta^{k i}$, because other roots of $\Phi_{\sigma^{k}}$ are less than $\beta^{k}$ in modulus. Our aim is to show that there are only finitely many matrices $M_{\sigma}^{k}=\left(a_{i j}\right)$. Indeed this implies that all entries of $M_{\sigma}$ are bounded by $\max \left\{a_{i j} \mid i, j \leq m\right\}$, since otherwise there is an entry of $M_{\sigma^{k}}$ larger than this bound. From $0 \leq \sum_{i} a_{i i}=c_{m-1} \leq m \beta^{k}$, we have $a_{i i}<m \beta^{k}$ and it suffices to show that $a_{i j}$ is bounded for $i \neq j$. Using

$$
\left(\begin{array}{c}
m \\
2
\end{array}\right) \beta^{2 k}>c_{m-2}=\sum_{i<j} a_{i j} a_{j i}-\sum_{i<j} a_{i i} a_{j j}
$$

and

$$
0<\sum_{i<j} a_{i i} a_{j j}=\frac{1}{2}\left(\left(\sum_{i} a_{i i}\right)^{2}-\sum_{i} a_{i i}^{2}\right) \leq \frac{m^{2}}{2} \beta^{2 k}
$$

we see that

$$
0 \leq a_{i j} a_{j i}<\sum_{i<j} a_{i j} a_{j i} \leq\left(\begin{array}{c}
m \\
2
\end{array}\right) \beta^{2 k}+\frac{m^{2}}{2} \beta^{2 k} \leq m^{2} \beta^{2 k}
$$

Since $a_{j i} \in \mathbb{N}$, we have the bound $a_{i j} \leq m^{2} B^{2 k}$. Thus we have $b_{i j} \leq m^{2} B^{2 k}$ where $M_{\sigma}=\left(b_{i j}\right)$ and the number of possible $\sigma(i)$ 's for each $i$ is bounded by the multinomial coefficient:

$$
\left(\begin{array}{c}
m^{3} B^{2 k} \\
m^{2} B^{2 k}, m^{2} B^{2 k}, \ldots, m^{2} B^{2 k}
\end{array}\right) \leq m^{m^{3} B^{2 k}} \leq m^{m^{3} B^{2(m-1)^{2}+2}}
$$

which gives the required bound.

Although Lemma 2.1 gives a bound for the number of substitutions whose Perron-Frobenius root is less than $B$, it is too large to be useful in practice. In the sequel, we deduce a practical estimate by the property of the Pisot number to narrow the range of computation. If $\sigma$ is a Pisot substitution of degree $d$ whose incidence matrix has a Pisot number $\beta$ as the Perron-Frobenius root, we have $\beta-(d-1)<\operatorname{Tr}\left(M_{\sigma}\right)<$ $\beta+(d-1)$. Thus it is meaningful to check the Pisot conjecture for irreducible substitutions whose incidence matrix $M_{\sigma}$ has small trace with a fixed degree. Our first result is the following proposition.

Proposition 2.2 Let $\sigma$ be a primitive, irreducible, cubic Pisot substitution with $\operatorname{Tr}\left(M_{\sigma}\right) \leq 2$. Then the spectrum of $\sigma$ is pure discrete.

Proof: Note that $\operatorname{Tr}\left(M_{\sigma}\right) \geq 0$ since $M_{\sigma}$ is a non-negative matrix. Let

$$
x^{3}-p x^{2}-q x-r, \quad \text { where } p, q, r \in \mathbb{Z}
$$

be the characteristic polynomial of $\sigma$. It is the minimal polynomial of a cubic Pisot number if and only if

$$
\max \left\{2-p-r, r^{2}-\operatorname{sign}(r)(1+p r)+1\right\} \leq q \leq p+r \text { and } r \neq 0
$$


holds (see [1]). We see $p=\operatorname{Tr}\left(M_{\sigma}\right) \geq 0$. Let

$$
M_{\sigma}:=\left(\begin{array}{ccc}
k_{1} & a & b \\
c & k_{2} & d \\
e & f & k_{3}
\end{array}\right)
$$

be the incidence matrix of the primitive substitution $\sigma$. Then the coefficients $p, q, r$ of the characteristic polynomial (2) can be written as

$$
\begin{aligned}
p & =k_{1}+k_{2}+k_{3} \text { and } p \geq 0, \\
q & =a c+b e+d f-k_{1} k_{2}-k_{2} k_{3}-k_{1} k_{3}, \\
r & =k_{1} k_{2} k_{3}+d c f+a d e-d f k_{1}-b e k_{2}-a c k_{3} .
\end{aligned}
$$

We claim that $a, b, c, d, e, f$ are not greater than $L$, where

$$
L:=\max \left\{q+k_{1} k_{2}+k_{2} k_{3}+k_{3} k_{1}, r-k_{1} k_{2} k_{3}+\max \left\{k_{1}, k_{2}, k_{3}\right\}\left(q+k_{1} k_{2}+k_{2} k_{3}+k_{1} k_{3}\right)\right\}
$$

using the idea of Lemma 2.1. Note that by the primitivity, none of six vectors $(a, b),(c, d),(e, f),(c, e)$, $(a, f),(b, d)$ is $(0,0)$. By symmetry, we only prove this bound (4) for $a$. Consider $q$ and $r$ as a linear polynomial on $a$. Then the leading coefficients are $c$ and $d e-c k_{3}$ and we see that either $c \neq 0$ or $d e-c k_{3} \neq 0$ holds. If $c \neq 0$, then the formula for $q$ gives $a \leq a c \leq q+k_{1} k_{2}+k_{2} k_{3}+k_{1} k_{3}$. If $c=0$ then, the formula for $q$ implies $b e+d f \leq q+k_{1} k_{2}+k_{2} k_{3}+k_{1} k_{3}$ and the formula for $r$ gives

$$
a \leq \text { ade } \leq r-k_{1} k_{2} k_{3}+d f k_{1}+b e k_{2} \leq r-k_{1} k_{2} k_{3}+\max \left\{k_{1}, k_{2}\right\}\left(q+k_{1} k_{2}+k_{2} k_{3}+k_{1} k_{3}\right) .
$$

So the claim follows. Since $k_{1}, k_{2}, k_{3}$ are non-negative integers, from (4) one can also deduce

$$
a, b, c, d, e, f \leq r+p\left(q+p^{2}\right) .
$$

In the case $p=0$ we easily have $q=r=1$ from (3). By using the bound of $a, b, c, d, e, f$, there are 6 substitutions matrices with the characteristic polynomial $x^{3}-x-1$. However, these matrices form a single orbit under the group $S_{3}$ which permutes the symbols, so that only one matrix with 2 substitutions needs to be checked. Moreover, there is a further symmetry which can be taken into account in order to avoid duplicate work. Let $\xi: \mathcal{A}^{*} \rightarrow \mathcal{A}^{*}$ be the 'mirror' map, i.e., $\xi\left(a_{1} a_{2} \ldots a_{n-1} a_{n}\right)=a_{n} a_{n-1} \ldots a_{2} a_{1}$. For a given substitution $\sigma$, we define $\bar{\sigma}$ by $\bar{\sigma}(a)=\xi(\sigma(a))$ for $a \in \mathcal{A}$. Then the dynamical systems $X_{\sigma}$ and $X_{\bar{\sigma}}$ are clearly isomorphic and we only have to compute one of them. By using this symmetry, the two substitutions of the $p=0$ case reduce to one, which turns out to be pure discrete. Next we study the case $p>0$.

Changing the order of letters, for $p=1$ we may assume that the incidence matrix $M_{\sigma}$ is of the form:

$$
\left(\begin{array}{lll}
1 & a & b \\
c & 0 & d \\
e & f & 0
\end{array}\right) \text { with } a \geq b
$$

and for $p=2$ we have

$$
\left(\begin{array}{lll}
2 & a & b \\
c & 0 & d \\
e & f & 0
\end{array}\right) \text { with } a \geq b, \text { or }\left(\begin{array}{lll}
1 & a & b \\
c & 1 & d \\
e & f & 0
\end{array}\right) \text { with } a \geq c
$$


For each case we can deduce bounds for $a, b, c, d, e, f$ from the inequality (4). The list of matrices satisfying these bounds is finite, but still contains some pairs which are equivalent under a permutation of the symbols. Only one matrix of each such pair is retained. For any substitution matrix $M$ in the list, there are only finitely many substitutions $\sigma$ with $M_{\sigma}=M$ (of which we keep only one per mirror pair), so that we obtain a finite list of irreducible Pisot substitutions to be checked. Then we use the 'potential overlap algorithm' of [3] and a new implementation [10] of the classical overlap algorithm [20, 21] to check all the substitutions in the list. Moreover, since the substitutions in the list are irreducible (Prop. 1.2.8 of [19]), we may check by the balanced pair algorithm [20] as well. Up to symbol relabelling and mirror symmetry as above, there are 7377 irreducible cubic Pisot unit substitutions, as given in Table 1, and all of them are pure discrete. The number of non-unit substitutions, especially with trace $p=2$, is much larger, and on average each computation is much harder. These non-unit cases could mostly be checked only with a new implementation [10] of the classical overlap algorithm in the GAP language [22], in which a special effort has been made to keep the memory requirements small. Also the non-unit substitutions turned out to be pure discrete. The results are summarized in Table 1.

\begin{tabular}{|c|c|c||c|c|l|}
\hline$p$ & $r$ & $q$ & $M_{\sigma}$ & $\sigma$ & Spectrum \\
\hline 0 & 1 & 1 & 1 & 1 & pure discrete \\
1 & 1 & $0,1,2$ & 12 & 61 & pure discrete \\
1 & 2 & 2,3 & 30 & 457 & pure discrete \\
2 & -1 & 1 & $1+4$ & $3+36$ & pure discrete \\
2 & 1 & $-1,0,1,2,3$ & $22+46$ & $1077+6199$ & pure discrete \\
2 & 2 & $0,1,2,3,4$ & $72+97$ & $10586+46348$ & pure discrete \\
2 & 3 & $3,4,5$ & $59+93$ & $65383+316532$ & pure discrete \\
\hline
\end{tabular}

Tab. 1: The columns labelled $M_{\sigma}$ and $\sigma$ contain the number of inequivalent substitution matrices and substitutions, respectively. In the trace 2 case, these numbers are split into the contributions from matrices with diagonal $(2,0,0)$ and $(1,1,1)$, respectively.

In principle, the above method of listing all cubic Pisot substitutions with a fixed trace can be used also for matrices $M_{\sigma}$ with $\operatorname{Tr}\left(M_{\sigma}\right)=3$. However, for this case there would be far too many substitutions to be checked, which currently seems beyond reach.

Experiments with an implementation of the balanced pair algorithm in GAP [10], similar to that of the classical overlap algorithm [20, 21], suggests that the latter has an advantage for more complex substitutions. This is likely due to the balanced pairs not only growing in number, but also in length, which requires more memory to store them, but especially also more work to compare them. The overlap types needed in the overlap algorithm, on the other hand, grow only in number, and each of them requires only a fixed (small) amount of memory.

\section{Substitution tilings in $\mathbb{R}^{2}$}

In this section, we check the pure discreteness of self-affine tilings constructed by Kenyon [14]. Before explaining his construction, we start with general notations. 
A tile in $\mathbb{R}^{2}$ is defined as a pair $T=(A, i)$ where $A=\operatorname{supp}(T)$ (the support of $T$ ) is a compact set in $\mathbb{R}^{2}$ which is the closure of its interior, and $i=l(T) \in\{1, \ldots, m\}$ is the color of $T$. We say that a set $P$ of tiles is a patch if the number of tiles in $P$ is finite and the tiles of $P$ have mutually disjoint interiors. A tiling of $\mathbb{R}^{2}$ is a set $\mathcal{T}$ of tiles such that $\mathbb{R}^{2}=\bigcup\{\operatorname{supp}(T): T \in \mathcal{T}\}$ and distinct tiles have disjoint interiors.

Let $\mathcal{A}=\left\{T_{1}, \ldots, T_{m}\right\}$ be a finite set of tiles in $\mathbb{R}^{2}$ such that $T_{i}=\left(A_{i}, i\right)$; we will call them prototiles. Denote by $\mathcal{P}_{\mathcal{A}}$ the set of non-empty patches. A substitution is a map $\Omega: \mathcal{A} \rightarrow \mathcal{P}_{\mathcal{A}}$ with a $2 \times 2$ expansive matrix $Q$ if there exist finite sets $\mathcal{D}_{i j} \subset \mathbb{R}^{2}$ for $i, j \leq m$ such that

$$
\Omega\left(T_{j}\right)=\left\{u+T_{i}: u \in \mathcal{D}_{i j}, i=1, \ldots, m\right\}
$$

with

$$
Q A_{j}=\bigcup_{i=1}^{m}\left(\mathcal{D}_{i j}+A_{i}\right) \text { for } j \leq m .
$$

Here all sets in the right-hand side must have disjoint interiors; it is possible for some of the $\mathcal{D}_{i j}$ to be empty.

We say that $\mathcal{T}$ is a substitution tiling if $\mathcal{T}$ is a tiling and $\Omega(\mathcal{T})=\mathcal{T}$ with some substitution $\Omega$. We say that $\mathcal{T}$ has finite local complexity (FLC) if $\forall R>0, \exists$ finitely many translational classes of patches whose support lies in some ball of radius $R$. A tiling $\mathcal{T}$ is repetitive if for any compact set $K \subset \mathbb{R}^{2}$, $\left\{t \in \mathbb{R}^{2}: \mathcal{T} \cap K=(t+\mathcal{T}) \cap K\right\}$ is relatively dense. A repetitive fixed point of a primitive substitution with FLC is called a self-affine tiling. Let $\lambda>1$ be the Perron-Frobenius eigenvalue of the substitution matrix $S$. Let $D=\left\{\lambda_{1}, \ldots, \lambda_{d}\right\}$ be the set of (real and complex) eigenvalues of $Q$. We say that $Q$ (or the substitution $\Omega$ ) fulfills the Pisot family property if, for every $\lambda \in D$ and every Galois conjugate $\lambda^{\prime}$ of $\lambda$, $\lambda^{\prime} \notin D$, then $\left|\lambda^{\prime}\right|<1$.

\subsection{Endomorphisms of free group}

Generalizing the idea of Dekking [8, 9], Kenyon [14] introduced a class of self-similar tilings generated by the endomorphisms of a free group over three letters $a, b, c$ :

$$
\begin{aligned}
& \theta(a)=b \\
& \theta(b)=c \\
& \theta(c)=c^{p} a^{-r} b^{-q}
\end{aligned}
$$

where $p, q \geq 0, r \geq 1$ are integers for which $x^{3}-p x^{2}+q x+r$ has exactly two roots $\lambda_{1}, \lambda_{2}$ with modulus greater than one (ii) The letters $a, b, c$ are identified with vectors $(1,1),\left(\lambda_{1}, \lambda_{2}\right),\left(\lambda_{1}^{2}, \lambda_{2}^{2}\right) \in \mathbb{R}^{2}$ respectively if $\lambda_{1}, \lambda_{2}$ are real numbers, and with $1, \lambda_{1}, \lambda_{1}^{2} \in \mathbb{C}$ if they are complex conjugates. The endomorphism $\theta$ acts naturally on the boundary word

$$
a b a^{-1} b^{-1}, a c a^{-1} c^{-1}, b c b^{-1} c^{-1},
$$

(ii) Kenyon [14] studied the cases when $\lambda_{i}$ are complex numbers. 


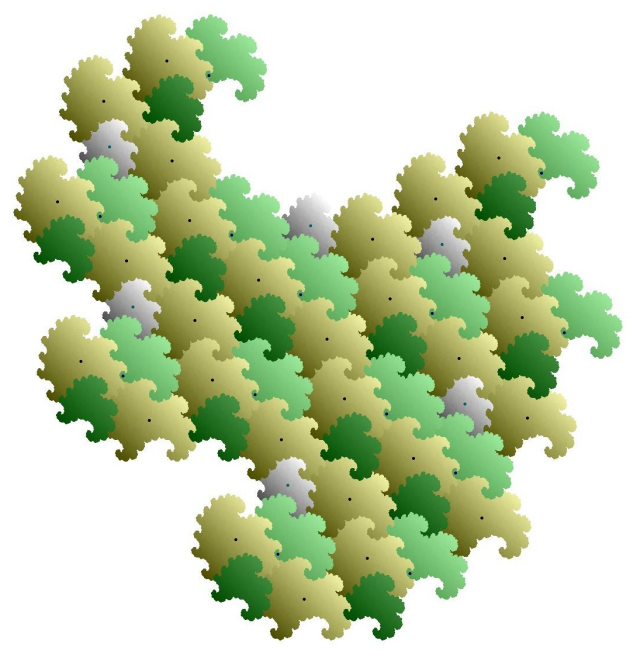

(a) Tiling

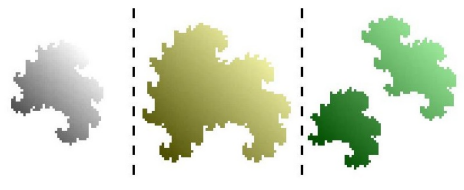

(b) Tiles

Fig. 1: Kenyon's tiling for $(p, q, r)=(2,1,1)$. The last tile is disconnected.

which represent three fundamental parallelograms, and gives a substitution rule on the parallelograms. The associated tile equations are

$$
\begin{aligned}
& Q T_{1}=T_{2} \\
& Q T_{2}=\left(\bigcup_{i=1}^{q}\left(T_{2}+p c-i b-r a\right)\right) \cup\left(\bigcup_{i=1}^{r}\left(T_{3}+p c-i a\right)\right) \\
& Q T_{3}=\left(\bigcup_{i=1}^{r}\left(T_{1}+p c-i a\right)\right) \cup\left(\bigcup_{i=1}^{p-1}\left(T_{2}+i c\right)\right)
\end{aligned}
$$

where $Q$ is either $\left(\begin{array}{cc}\lambda_{1} & 0 \\ 0 & \lambda_{2}\end{array}\right)$ or $\lambda_{1}$ depending on whether $\lambda_{1}$ is real or complex, respectively.

It is known [15, 16] that if the expansion map $Q$ of a self-affine tiling in $\mathbb{R}^{2}$ is diagonalizable and the tiling has pure discrete dynamical spectrum, then $Q$ should fulfill the Pisot family property. So we are interested in considering self-affine tilings with the Pisot family property on the expansion map $Q$. For this construction, we require that two roots of the polynomial $x^{3}-p x^{2}+q x+r$ are greater than one, and one root is smaller than one in modulus. In this case, we can note that there are no roots on the unit circle. We adapt the Schur-Cohn criterion (see [1, Theorem 2.1] or [17, Chap.10, Th.43, 1]), which says that the number of roots within the unit circle coincides with the number of sign changes of the following sequence:

$$
1, \Delta_{1}, \Delta_{2}, \Delta_{3}
$$


where

$$
\begin{aligned}
& \Delta_{1}=-\left|\begin{array}{ll}
1 & r \\
r & 1
\end{array}\right|=(r-1)(r+1), \\
& \Delta_{2}=\left|\begin{array}{rrrr}
1 & -p & r & 0 \\
0 & 1 & q & r \\
r & q & 1 & 0 \\
0 & r & -p & 1
\end{array}\right|=-\left(p r+q-r^{2}+1\right)\left(p r+q+r^{2}-1\right) \text {, } \\
& \Delta_{3}=-\left|\begin{array}{rrrrrr}
1 & -p & q & r & 0 & 0 \\
0 & 1 & -p & q & r & 0 \\
0 & 0 & 1 & -p & q & r \\
r & q & -p & 1 & 0 & 0 \\
0 & r & q & -p & 1 & 0 \\
0 & 0 & r & q & -p & 1
\end{array}\right| \\
& =(p-q-r-1)(p+q-r+1)\left(p r+q+r^{2}-1\right)^{2},
\end{aligned}
$$

if all entries are non-zero. Notice that it cannot be that $p=q=0$, otherwise all the roots of the polynomial have the same modulus. Thus from $p, q \geq 0$ and $r \geq 1$, pr $+q+r^{2}-1>0$. So the signs come from

$$
1,(r-1)(r+1),-\left(p r+q-r^{2}+1\right),(p-q-r-1)(p+q-r+1)
$$

The last term is not zero, since \pm 1 cannot be a root of $x^{3}-p x^{2}+q x+r$. When $r=1$, the second term vanishes and the third term is negative (because $p$ or $q$ is positive). Since the roots of the polynomial are continuous with respect to the coefficients, the small perturbation of $r$ does not change the number of roots inside/outside of the unit circle. Therefore we may assume that the second coefficients are non-zero and use the Schur-Cohn criterion (c.f. [1]). As a result, the number of zeroes within the unit circle is 1 when $(p-q-r-1)(p+q-r+1)<0$ and it is 2 when $(p-q-r-1)(p+q-r+1)>0$. If $r>1$, then the second term is positive. In this case, applying the small perturbation argument when the third term vanishes, the number of zeroes within the unit circle is 1 when $(p-q-r-1)(p+q-r+1)<0$ and 0 or 2 when $(p-q-r-1)(p+q-r+1)>0$. Overall we obtain a unified conclusion that the number of zeroes within the unit circle is 1 if and only if $(p-q-r-1)(p+q-r+1)<0$. Our tiling exists when $|p-r|<q+1$.

For $\max \{p, q, r\} \leq 3$, there are 34 cases:

$$
\begin{gathered}
\{0,1,1\},\{0,2,1\},\{0,2,2\},\{0,3,1\},\{0,3,2\},\{0,3,3\},\{1,0,1\},\{1,1,1\},\{1,1,2\}, \\
\{1,2,1\},\{1,2,2\},\{1,2,3\},\{1,3,1\},\{1,3,2\},\{1,3,3\},\{2,0,2\},\{2,1,1\},\{2,1,2\}, \\
\{2,1,3\},\{2,2,1\},\{2,2,2\},\{2,2,3\},\{2,3,1\},\{2,3,2\},\{2,3,3\},\{3,0,3\},\{3,1,2\}, \\
\{3,1,3\},\{3,2,1\},\{3,2,2\},\{3,2,3\},\{3,3,1\},\{3,3,2\},\{3,3,3\}
\end{gathered}
$$

We determined the spectral type of the tiling dynamical systems except for the case $\{3,0,3\}$. All computed systems admit an overlap coincidence, and thus have a pure discrete spectrum. In the remaning case $\{3,0,3\}$, the tiles are very thin, so that the number of initial overlaps seems to be beyond the capability of our program. 


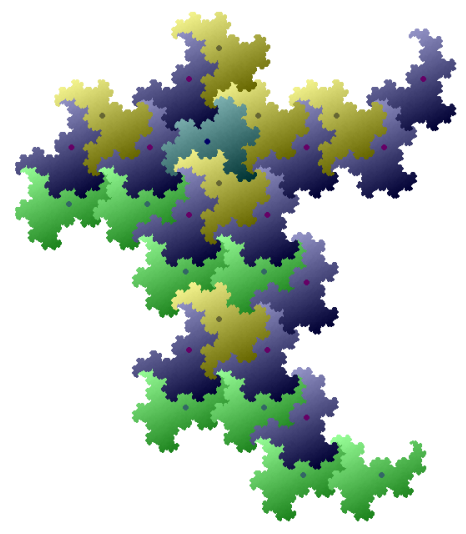

Fig. 2: Fractal chair tiling

\section{Examples with non pure discrete spectrum}

In this section, we wish to give two intriguing examples of self-affine tilings whose dynamical spectrum is not pure discrete. Both tiling dynamics are not weakly mixing and therefore there exist non-trivial eigenvalues of their translation actions.

Bandt discovered a non-periodic tiling in [6], whose setting comes from crystallographic tiles, and called it the fractal chair tiling. The tile satisfies the following set equation

$$
-I \omega \sqrt{3} A=A \cup(A+1) \cup(\omega A+\omega)
$$

where $\omega=(1+\sqrt{-3}) / 2$ is the 6-th root of unity. It is called 3-rep-tile, because it is a non-overlapping union of three similar contracted copies, i.e., the associated iterated function system satisfies the open set condition [6]. Applying the substitution rule, we obtain a tiling of the plane by six tiles $T_{i}=\omega^{i} A(i=$ $0,1,2,3,4,5)$ and their translates as given in Figure 2 This tiling is non-periodic, i.e., the only translation which sends the tiling exactly to itself is the zero vector. We can confirm that this tiling is repetitive and the corresponding expansion map satisfies the Pisot family property. So we can apply the potential overlap algorithm from [3]. Our program shows that the fractal chair tiling is not purely discrete. The overlap graph with multiplicity contains a strongly connected component of spectral radius 3 , being equal to the spectral radius of the substitution matrix, which does not lead to a coincidence. In fact, the existence of such a component is shown in [3] to be equivalent to having non-pure discrete spectrum. Geometrically this means that there is a finite set $C$ of overlaps not containing any coincidence, which is mapped to itself under the substitution. We call such a set $C$ a non-coincident component. We visualize in Figure 3 how each overlap in the non-coincident component does not lead to a coincidence. Each figure represents an overlap of two fractal chair tiles, and the support of the overlap is depicted in thick color. The destinations of outgoing arrows show that we obtain three overlaps from each overlap by substitution. 


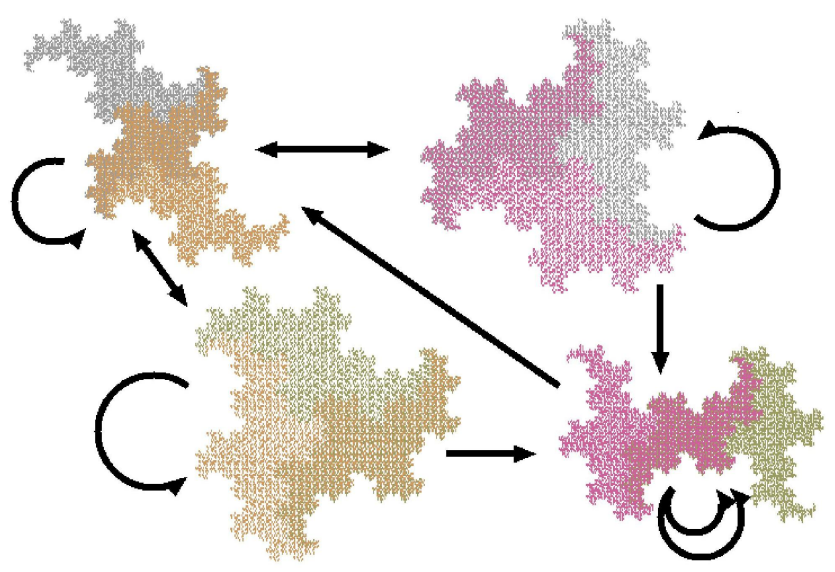

Fig. 3: Non-coincident component of fractal chair tiling

As another example, the following substitution

$$
\begin{aligned}
& 1 \rightarrow 1241224 \\
& 2 \rightarrow 1224 \\
& 3 \rightarrow 1243334 \\
& 4 \rightarrow 124334
\end{aligned}
$$

with the characteristic polynomial $\left(x^{2}-3 x+1\right)\left(x^{2}-6 x+1\right)$ arose in the study of self-inducing 4 interval exchanges by P. Arnoux, S. Ito and M. Furukado [13]. As it is a Pisot substitution, the natural suspension tiling satisfies the Pisot family condition and our potential overlap algorithm readily applies. It is of interest to check this non-weakly mixing system, as it is shown by [5] that a generic interval exchange transformation which is not a rotation is weakly mixing. Our algorithm shows that its suspension tiling dynamics is not pure discrete. Although there are several known reducible Pisot substitutions which are not pure discrete, this example is noteworthy in the sense that the non-coincident component is much 
more intricate than in other known examples. We describe it by another substitution on the 12 letters:

$$
\begin{aligned}
a & \rightarrow \text { afdeEafdc, } \\
b & \rightarrow f B C F b c f d e E, \\
c & \rightarrow \text { afdc, } \\
A & \rightarrow \text { AFDEeAFDC, } \\
B & \rightarrow \text { FbcfBCFDEe, } \\
C & \rightarrow \text { AFDC, } \\
d & \rightarrow \text { FbcfdeE, } \\
D & \rightarrow f B C F D E e, \\
e & \rightarrow a f d e E e, \\
E & \rightarrow A F D E e E, \\
f & \rightarrow f B C, \\
F & \rightarrow F b c
\end{aligned}
$$

The associated tile equation of this substitution gives the non-coincident component of the suspension tiling corresponding to (7). In other words, (8) is the exact analogue of Figure 3, once we associate the intervals of canonical lengths given by the left eigenvector of the substitution matrix. Since the original tiling is a factor of this new suspension tiling, the system is again not pure discrete.

The above two substitution examples have reducible characteristic polynomials. So the assumption of the Pisot substitution conjecture in higher dimensions of [4] does not hold.

\section{Acknowledgment}

We are grateful to P. Arnoux, Sh. Ito and M. Furukado for permitting us to include the last example for the study of its spectrum. This work was supported by the National Research Foundation of Korea(NRF) Grant funded by the Korean Government(MSIP)(2014004168), the Japanese Society for the Promotion of Science (JSPS), Grant in aid 21540012, and the German Research Foundation (DFG) through the CRC 701 Spectral Structures and Topological Methods in Mathematics. The first author is partially supported by the TÁMOP-4.2.2.C-11/1/KONV-2012-0001 project. (The project is implemented through the New Hungary Development Plan, co-financed by the European Social Fund and the European Regional Development Fund.) The third author is grateful for the support of Korea Institute for Advanced Study(KIAS) for this research.

\section{References}

[1] S. Akiyama and N. Gjini. Connectedness of number theoretic tilings. Discrete Math. Theor. Comput. Sci., 7(1): 269-312 (electronic), 2005. ISSN 1365-8050.

[2] S. Akiyama and J.-Y. Lee. http://math.tsukuba.ac.jp/ akiyama/research1.html, http://newton.kias.re.kr/ jeongyup/research/. 
[3] S. Akiyama and J.-Y. Lee. Algorithm for determining pure pointedness of self-affine tilings. Adv. Math., 226 (4):2855-2883, 2011. ISSN 0001-8708. doi: 10.1016/j.aim.2010.07.019. URLhttp://dx.doi.org/10. $1016 / j . a i m .2010 .07 .019$

[4] S. Akiyama, M. Barge, V. Berthé, J.-Y. Lee, and A. Siegel. On the pisot substitution conjecture. In Aperiodic order(ed. J. Kellendonk, D. Lenz, J. Savinien), Progress in Mathematics. Birkhauser, Submitted.

[5] A. Avila and G. Forni. Weak mixing for interval exchange transformations and translation flows. Ann. of Math., 165(2):637-664, 2007.

[6] C. Bandt. Self-similar tilings and patterns described by mappings. In The Mathematics of Long-Range Aperiodic Order, ed. R.V. Moody, volume 489 of NATO ASI series C, pages 45-83. Kluwer, Dordrecht, 1997.

[7] A. Clark and L. Sadun. When size matters: subshifts and their related tiling spaces. Ergodic Theory Dynam. Systems, 23(4):1043-1057, 2003. ISSN 0143-3857. doi: 10.1017/S0143385702001633. URL http://dx. doi.org/10.1017/S0143385702001633

[8] F. M. Dekking. Recurrent sets. Adv. in Math., 44(1):78-104, 1982.

[9] F. M. Dekking. Replicating superfigures and endomorphisms of free groups. J. Combin. Theory Ser. A, 32(3): $315-320,1982$.

[10] F. Gähler. http://www.math.uni-bielefeld.de/ gaehler/gap/tilings/.

[11] J. C. Holladay and R. S. Varga. On powers of non-negative matrices. Proc. Amer. Math. Soc., 9:631-634, 1958.

[12] M. Hollander and B. Solomyak. Two-symbol pisot substitutions have pure discrete spectrum. Ergod. Th. \& Dynam. Syst., 23:533-640, 2003.

[13] S. Ito. Substitutions from rauzy induction on 4-interval exchange transformations and quasi-periodic tilings. from the talk slides of the lecture delivered in RIMS Kyoto, 2010.

[14] R. Kenyon. The construction of self-similar tilings. Geometric and Funct. Anal., 6:471-488, 1996.

[15] J.-Y. Lee and B. Solomyak. Pure point diffractive substitution delone sets have the meyer property. Discrete Comput. Geom., 39(1-3):319-338, 2008. ISSN 0179-5376. doi: 10.1007/s00454-008-9054-1. URL http: //dx.doi.org/10.1007/s00454-008-9054-1.

[16] J.-Y. Lee and B. Solomyak. Pisot family self-affine tilings, discrete spectrum, and the meyer property. Discrete and Continuous Dynamical Systems - A, 32:935-959, 2012.

[17] M. Marden. Geometry of polynomials, volume 3. American Mathematical Society, Providence, R.I., 1966.

[18] V. Pták. On a combinatorial theorem and its application to nonnegative matrices. Czechoslovak Math. J, 8(83): 487-495, 1958.

[19] N. Pytheas Fogg. Substitutions in Dynamics, Arithmetics and Combinatorics, volume 1794 of Lecture Notes in Mathematics. Springer-Verlag, 2002. Ed. by V. Berthé and S. Ferenczi and C. Mauduit and A. Siegel.

[20] V. F. Sirvent and B. Solomyak. Pure discrete spectrum for one-dimensional substitution systems of Pisot type. Canad. Math. Bull., 45(4):697-710, 2002. Dedicated to Robert V. Moody.

[21] B. Solomyak. Dynamics of self-similar tilings. Ergod. Th. \& Dynam. Sys., 17:695-738, 1997.

[22] The GAP Group. Gap - groups, algorithms, and programming, version 4.7.2, http: //www.gap-system.org+. 2013. 